\title{
Risk of neuropsychiatric disorders in offspring of COVID-19-infected pregnant women and nutritional intervention
}

\author{
Kenji Hashimoto ${ }^{1}$ (1)
}

Received: 2 May 2020 / Accepted: 29 May 2020 / Published online: 2 June 2020

c) Springer-Verlag GmbH Germany, part of Springer Nature 2020

After the detection of the novel coronavirus disease (COVID-19) in December 2019 in Wuhan city, Hubei province, China, individuals infected with COVID-19 are markedly increasing in the world. On March 11, 2020, the Word Health Organization declares COVID-19 outbreak a pandemic. The ongoing COVID-19 outbreak is a public health emergency worldwide. However, there are currently no approved drugs for COVID-19 although clinical trials of a number of candidates are underway [1].

On February 12, 2020, Chen et al. [2] reported the clinical characteristics of COVID-19 in nine pregnant women with laboratory-confirmed COVID-19 pneumonia and intrauterine vertical transmission potential of COVID-19 at the Zhongnan Hospital of Wuhan University, China. All patients underwent caesarean sections in the third trimester. The range of gestational weeks at admission was 36 weeks to 39 weeks plus 4 days. Gestational age of the four patients was before 37 weeks. Nine livebirths were recorded without fetal death, neonatal death, or neonatal asphyxia observed in the newborn babies. Thus, there is currently no evidence showing intrauterine infection caused by vertical transmission in women who develop COVID-19 pneumonia in the late stages of pregnancy [2]. However, further study using a large sample size is needed.

Epidemiological studies suggest that maternal immune activation (MIA) plays a role in the etiology of neuropsychiatric disorders such as autism spectrum disorder (ASD) and schizophrenia. Maternal infectious pathogens and inflammation have been associated with these neuropsychiatric disorders. C-reactive protein (CRP) is a well-established blood biomarker of inflammation from both infectious and

Communicated by Andrea Schmitt.

Kenji Hashimoto

hashimoto@faculty.chiba-u.jp

1 Division of Clinical Neuroscience, Chiba University Center for Forensic Mental Health, Chiba 260-8670, Japan noninfectious exposures. Among the nine pregnant women infected with COVID-19, six displayed elevated concentration $(>10 \mathrm{mg} / \mathrm{L})$ of CRP, which indicated severe inflammation [2].

A national birth study in Finland suggested that high levels of CRP during pregnancy are significantly associated with ASD in offspring [3]. A significant elevated risk of $43 \%$ is observed in maternal early gestational CRP levels in the highest quintile ( $>58.4 \mathrm{mg} / \mathrm{L}$ ) compared with the lowest quintile (1.0-9.2 mg/L) [3]. Furthermore, a meta-analysis showed that higher maternal CRP levels were significantly associated with schizophrenia in offspring (odds ratio 1.31, 95\% confidence interval (CI) 1.11-1.55, standard mean differences $=0.15,95 \% \mathrm{CI} 0.06-0.24, P<0.01)$ [4]. In contrast, there is still a lot of debate on the relationship of CRP levels in pregnancy and neuropsychiatric disorders in offspring. It is widely recognized that MIA, such as elevated maternal CRP, may play a significant role in the development of these neuropsychiatric disorders in offspring although further study is needed.

There are increasing interests in the potential benefit of early intervention by safe anti-inflammatory nutrition since MIA including maternal infection which plays a crucial role in the development of neuropsychiatric disorders [5, 6]. Consumption of broccoli is known to bring a number of health benefits, including reducing the risk of cancer and inflammatory diseases. Sulforaphane is an organosulfur compound derived from a glucosinolate precursor glucoraphanin, which is found in cruciferous vegetables such as broccoli sprout (Fig. 1) [5, 6]. Glucoraphanin is metabolized to sulforaphane by the plant enzyme myrosinase, active during chewing, or gut microbiota. Sulforaphane is well recognized to have potent anti-inflammatory and anti-oxidant effects $[5,6]$.

Previously, we reported that dietary intake of glucoraphanin food pellet during juvenile and adolescence could attenuate the development of schizophrenia-like behaviors in mice after repeated administration of phencyclidine [7]. In rodents, MIA using polyriboinosinic-polyribocytidylic 
Fig. 1 Potential intervention by dietary intake of antiinflammatory nutrition on the development of neuropsychiatric disorders in offspring after maternal infection of COVID19. Maternal immune activation (MIA) by COVID-19 infection causes inflammatory events in pregnant women with COVID19 infection, resulting in higher CRP levels in blood. Given the role of MIA in neuropsychiatric disorders such as ASD and schizophrenia, follow-up investigation of pregnant women infected with COVID19 and their children is needed. For example, dietary intake of anti-inflammatory nutrition (i.e., sulforaphane and its precursor glucoraphanin) may reduce the risk of neuropsychiatric disorders in offspring after maternal infection of COVID-19 $\begin{array}{lll}\text { Pregnancy } & \text { Childhood } & \text { Adolescence \& }\end{array}$

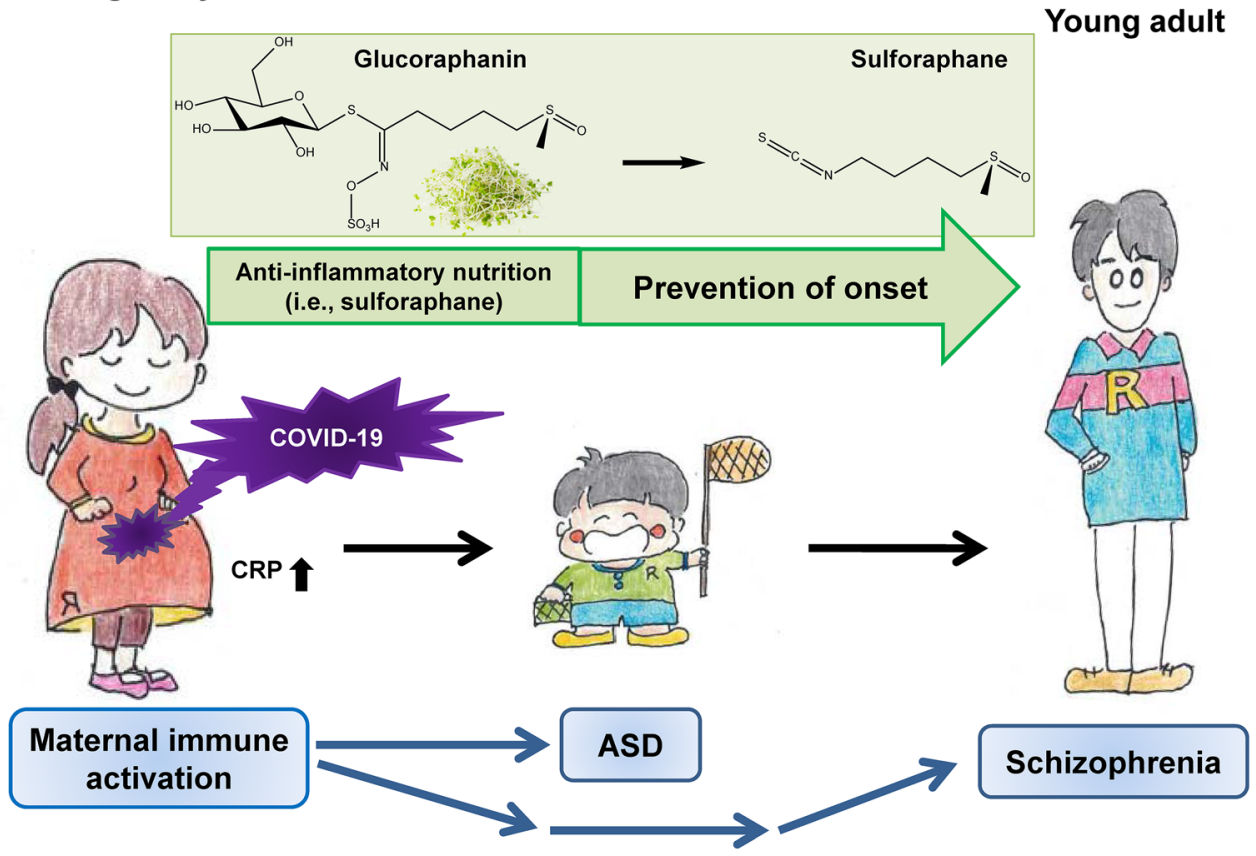

acid [poly (I:C)], a toll-like receptor 3 agonist, produces ASD-like and schizophrenia-like behaviors in offspring. Therefore, MIA model using poly (I:C) has been widely used as an animal model for neurodevelopmental disorders. We reported that dietary intake of glucoraphanin food pellet during juvenile and adolescence could attenuate the development of schizophrenia-like behavior in adult offspring after MIA [8]. Interestingly, dietary intake of glucoraphanin food pellet during pregnancy and lactation could attenuate the development of ASD-like and schizophrenia-like behaviors in juvenile and adult offspring after MIA [9]. Interestingly, Singh et al. [10] demonstrated that dietary intake of sulforaphane caused substantial declines of Aberrant Behavior Checklist and Social Responsiveness Scale scores in young men $(n=29)$ with ASD. A subsequent follow-up study showed that many parents and caregivers had positive effects of sulforaphane, both during the intervention study and the ensuing three years [11]. Beneficial effects of sulforaphane are of great interest although future studies using a large sample size should address its potential benefits for the prenatal prevention of ASD as well as for the delay in the early prevention of young children with ASD $[10,11]$. Collectively, it is likely that dietary intake of glucoraphanin food pellet could produce potent prophylactic effects in the development of neuropsychiatric disorders in rodents $[6,7]$.

In conclusion, the number of pregnant women infected with COVID-19 is expected to increase in the world [12]. It is very important to prevent the infection of COVID-19 in women during pregnancy. Given the risk of MIA in neuropsychiatric disorders such as ASD and schizophrenia, follow-up investigations of pregnant women infected with
COVID-19 and their children will be necessary to ascertain the risk of neuropsychiatric disorders, such as ASD and schizophrenia, in offspring. Furthermore, dietary intake of anti-inflammatory nutrition (i.e., sulforaphane and its precursor glucoraphanin) in pregnant women with COVID-19 infection and their children may reduce the risk of neuropsychiatric disorders such as ASD and schizophrenia (Fig. 1). Future randomized, double-blind, placebo-control study is needed to ascertain whether supplementation of sulforaphane or its precursor glucoraphanin in pregnant women with COVID-19 infection or their children could reduce the onset of neuropsychiatric disorders.

Acknowledgements This work was supported in part by a Grant-in-Aid for Scientific Research (B), Culture, Sports, Science and Technology, Japan (to K.H., 17H04243), the Strategic Research Program for Brain Sciences from Japan Agency for Medical Research and Development, AMED (to K.H., JP20dm0107119), and SENSHIN Medical Research Foundation, Japan (to K.H.). The author thanks Ms. Yuko Fujita for preparation of the figure.

Funding Dr. Hashimoto received speaker's honoraria from Murakami Farm (Tokyo, Japan) which sells sulforaphane-rich vegetable.

\section{References}

1. Zhang J, Xie B, Hashimoto K (2020) Current status of potential therapeutic candidates for the COVID-19 crisis. Brain Behav Immun. https://doi.org/10.1016/j.bbi.2020.04.046

2. Chen H, Guo J, Wang C, Luo F, Yu X, Zhang W, Li J, Zhao D, Xu D, Gong Q, Liao J, Yang H, Hou W, Zhang Y (2020) Clinical characteristics and intrauterine vertical transmission potential 
of COVID-19 infection in nine pregnant women: a retrospective review of medical records. Lancet 395:809-815

3. Brown AS, Sourander A, Hinkka-Yli-Salomaki S, McKeague IW, Sundvall J, Surcel HM (2014) Elevated maternal C-reactive protein and autism in a national birth cohort. Mol Psychiatry 19:259-264

4. Zhang J, Luo W, Huang P, Peng L, Huang Q (2018) Maternal $\mathrm{C}$-reactive protein and cytokine levels during pregnancy and the risk of selected neuropsychiatric disorders in offspring: a systematic review and meta-analysis. J Psychiatr Res 105:86-94

5. Hashimoto K (2018) Essential role of Keap1-Nrf2 signaling in mood disorders: overview and future perspective. Front Pharmacol 9:1182

6. Hashimoto K (2019) Recent advances in the early intervention in schizophrenia: future direction from preclinical findings. Curr Psychiatry Rep 21:75

7. Shirai Y, Fujita Y, Hashimoto R, Ohi K, Yamamori H, Yasuda Y, Ishima T, Suganuma H, Ushida Y, Takeda M, Hashimoto K (2015) Dietary intake of sulforaphane-rich broccoli sprout extracts during juvenile and adolescence can prevent phencyclidine-induced cognitive deficits at adulthood. PLoS ONE 10:e0127244
8. Matsuura A, Ishima T, Fujita Y, Iwayama Y, Hasegawa S, Kawahara-Miki R, Maekawa M, Toyoshima M, Ushida Y, Suganuma H, Kida S, Yoshikawa T, Iyo M, Hashimoto K (2018) Dietary glucoraphanin prevents the onset of psychosis in the adult offspring after maternal immune activation. Sci Rep 8:2158

9. Fujita Y, Fujita A, Ishima T, Hirai A, Suzuki S, Suganuma H, Hashimoto K (2020) Dietary intake of glucoraphanin during and lactation prevents the behavioral abnormalities in the offspring after maternal immune activation. Neuropsychopharmacol Rep. https://doi.org/10.1002/npr2.12112(in press)

10. Singh K, Connors SL, Macklin EA, Smith KD, Fahey JW, Talalay P, Zimmerman AW (2014) Sulforaphane treatment of autism spectrum disorder (ASD). Proc Natl Acad Sci USA 111:15550-15555

11. Lynch R, Diggins EL, Conners SL, Zimmerman AW, Singh K, Liu H, Talalay P, Fahey JW (2017) Sulforaphane from broccoli reduces symptoms of autism: a follow-up case series from a randomized double-blind study. Glob Adv Health Med 6:2164957X17735826

12. Yang Z, Wang M, Zhu Z, Liu Y (2020) Coronavirus disease 2019 (COVID-19) and pregnancy: a systematic review. J Matern Detal Neonatal Med. https://doi.org/10.1080/14767058.2020.1759541 